\title{
Lamotrigine Is Neuroprotective in the Energy Deficiency Model of MPTP Intoxicated Mice
}

\author{
EMMANUELLE LAGRUE, SYLVIE CHALON, SYLVIE BODARD, ELIE SALIBA, PIERRE GRESSENS, \\ AND PIERRE CASTELNAU
}

\begin{abstract}
Inserm, U619 [E.L., S.C., S.B., E.S., P.C.], Tours, F-37000 France; Université François-Rabelais [E.L., SC, E.S., P.C.], Tours, F-37000
France; CHRU Tours [E.L., E.S., P.C.], Hôpital Clocheville, Tours, F-37000 France; Inserm, U676 [P.G.], Paris, F-75019, France;

Université Paris 7 Denis-Diderot [P.G.], Faculté de médecine Xavier Bichat, IFR02, Paris, F-75019, France; Hôpital Robert Debré

[P.G.], Paris, F-75019, France
\end{abstract}

\begin{abstract}
The 1-methyl-4-phenyl-1,2,3,6-tetrahydropyridine (MPTP) inhibits the mitochondrial complex I of the respiratory chain. This results in ATP and ion homeostasis disturbances, which lead to selective death of the substantia nigra dopaminergic neurons. Well known as a Parkinson's disease model, the MPTP animal model also provides a potential paradigm of the energy deficiencies found in childhood. In these conditions, anticonvulsants may provide neuroprotection by limiting cellular energy consumption. We tested valproate, topiramate and lamotrigine in the MPTP mouse model. Dopamine transporter (DAT) density was assessed by quantitative autoradiography, tyrosine hydroxylase $(\mathrm{TH})$ was evaluated by immunohistochemistry and dopamine (DA) levels by HPLC-ED whereas neuronal apoptosis was monitored through active caspase-3. Expectedly, the DAT density, TH immunoreactive neurons and DA content in the MPTP group were respectively reduced to $51 \%, 40 \%$ and $26 \%$ versus control animals. Unlike valproate and topiramate, lamotrigine provided a significant neuroprotection against MPTP in maintaining these levels at $99 \%, 74 \%$ and $58 \%$ respectively and reducing the induced apoptosis. Altogether, the data indicate that lamotrigine limits dopaminergic neuronal death in the substantia nigra and promotes striatal dendrites sprouting. Lamotrigine, a widely used and well-tolerated molecule in young patients, could represent a valuable adjuvant therapy in various energy deficiency conditions during childhood. (Pediatr Res 62: 14-19, 2007)
\end{abstract}

$\mathrm{E}$ vidences from degenerative and metabolic encephalopathies studies indicate that the striatum and the substantia nigra $(\mathrm{SN})$ are vulnerable structures in the brain, especially during pre and postnatal brain development $(1,2)$. A partial or total destruction of this striato-nigral dopaminergic network is found in several conditions occurring in childhood such as iron or copper metabolism abnormalities (1), organic acidurias (3), urea cycle disorders, various mitochondrial energy production deficiencies $(1,4)$, or hypoxia-ischemia $(2,5)$. All these conditions witness the vulnerability of this particular deep nuclei network involved in multiple motor, sensory and cognitive functions.

Received October 23, 2006; accepted March 5, 2007.

Correspondence: Pierre Castelnau, M.D., Ph.D., Neuropédiatrie et Inserm U619, Université de Tours, Hôpital d'Enfants Gatien de Clocheville, 49 Bd Béranger, 37044 Tours cedex 01, France, Phone: 33 (0)2 47478200 - Fax: 33 (0)2 47478250; E-mail: castelnau@med.univ-tours.fr

E.L. was supported by a fellowship from Journées de Neurologie de Langue Française.
These basal ganglia share a common susceptibility to energetic stress $(6,7)$, which can be studied experimentally using mitochondrial inhibitors (8). Once converted into MPP+, the neurotoxin 1-methyl-4-phenyl-1,2,3,6-tetrahydropyridine (MPTP) inhibits the mitochondrial electron transport chain at complex I (NADH-ubiquinone oxidoreductase) resulting in decreased oxygen consumption and ATP production and ion homeostasis disturbances which, together, lead to neuronal cell death (9). This toxin depletes dopaminergic neurons in the $\mathrm{SN}$ and causes neuronal degeneration of the nigrostriatal pathway in animals and humans (9).

Several neuroprotection studies were performed in this MPTP mouse model using: energy sparing drugs; free radicals scavengers; anti-glutamatergic drugs; anti-apoptotic molecules and neurotrophines (10-12).

Anticonvulsants share several cell protective properties such as ATP-dependent ion channel inhibition, glutamate release inhibition and GABA release stimulation. We chose to test in the MPTP mouse model, anticonvulsants that operate on a large number of the pathologic ways described above and which might spare energy through ion channels inhibition. ATP dependent ion channels inhibition may indeed protect neurons as it adapts the cellular metabolism to the limited ATP availability during the energetic distress (13). Moreover, current anticonvulsants carry now a reasonable tolerance profile in humans even in young children. Therefore, anticonvulsants provide good candidates for neuroprotection assays especially in energy stress conditions in childhood.

Among them, we tested topiramate (TOP), valproate (VPA) and lamotrigine (LMT), each of which recapitulates these properties (14-16). None of these drugs activate cytochrome $\mathrm{P} 450$ or monoamine oxidase B that interferes with MPP+ metabolism thereby reducing MPTP toxicity (9). TOP has never been tested in the MPTP mice model. This relatively new anticonvulsant exhibits neuroprotective properties, which were reported in other conditions such as status epilepticus

Abbreviations: ADCC, ATP dependent calcium channel; AU, arbitrary unit; DA, dopamine; DAT, dopamine transporter; LMT, lamotrigine; MPTP, 1-methyl-4-phenyl 1,2,3,6 tétrahydropyridine; RT, room temperature; SN, substantia nigra; SNpc, substantia nigra pars compacta; $\mathbf{T H}$, tyrosine hydroxylase; TOP, topiramate; VPA, valproate 
and hypoxia-ischemia $(17,18)$. VPA, tested by Melamed et al., did not show significant neuroprotection (19). In contrast, LMT could have neuroprotective properties when associated to MPTP administration (20). However, the mechanism by which LMT provides this effect remains poorly understood.

In the present study, we examined, through tyrosine hydroxylase (TH) and dopamine transporter (DAT), two specific dopaminergic markers, the neuroprotective properties of VPA, TOP and LMT on MPTP-induced neurotoxicity in mice. As LMT showed marked neuroprotective properties in this model, we subsequently focused on this well tolerated anticonvulsant to clarify its mechanisms of action using apoptosis and dopamine (DA) content analysis.

\section{MATERIALS AND METHODS}

Animals. All experiments were performed on consanguineous male C57/ B16N@Rj mice (5 wk old, average weight: $19 \pm 1 \mathrm{~g}$, CERJ, France) in accordance with the European Community Commission guidelines (86/609/ EEC). The mice were kept under controlled conditions on a $12 \mathrm{~h}$ light/dark cycle with food and water ad libitum. Since mature levels of monoamine oxidase B are required to convert MPTP into its active toxic form MPP+, younger animals were not used in the present study.

Anticonvulsant treatments. Five groups ( $n=6$ per group) were constituted for DAT and TH study: control (saline p.o. and i.p.), MPTP (saline p.o. - MPTP i.p.), VPA-MPTP (VPA p.o. - MPTP i.p.), TOP-MPTP (TOP p.o. MPTP i.p.) and LMT-MPTP (LMT p.o. - MPTP i.p.). Each group was treated with the corresponding anticonvulsant twice a day p.o. from day 1 (D1) to day 7 (D7). The cumulative dose was $100 \mathrm{mg} / \mathrm{kg} / \mathrm{d}$ for VPA (Depakine ${ }^{\circledR}$, SanofiSynthelabo, France) and TOP (Epitomax ${ }^{\circledR}$, Janssen-Cilag, France) and 40 $\mathrm{mg} / \mathrm{kg} / \mathrm{d}$ for LMT Lamictal $^{\circledR}$, GlaxoSmithKline, France). Each anticonvulsant was dissolved in saline to obtain a final $200 \mu \mathrm{L}$ volume of oral administration. Each mice was weighted daily.

MPTP intoxication. The mice were intoxicated with 4 administrations of MPTP (12.5 mg/kg) i.p. at $1 \mathrm{~h}$ intervals on D3. MPTP (Sigma Chemical Co., France) was dissolved in $0.9 \%$ sodium chloride to a final concentration of 2.5 $\mathrm{mg} / \mathrm{mL}$ (100 $\mu \mathrm{L}$ injection/20g mouse body weight). The control mice only received saline.

DAT autoradiographic studies. The mice were killed by cervical dislocation $7 \mathrm{~d}$ after MPTP intoxication (i.e., $3 \mathrm{~d}$ after the end of the anticonvulsant treatment). The brains were rapidly removed, frozen $\left(-35^{\circ} \mathrm{C}\right)$, subsequently cut into $20 \mu \mathrm{m}$ coronal sections (Reichert-Jung Cryocut CM3000 Leica, France) and stored at $-80^{\circ} \mathrm{C}$.

For binding studies, 3 sections/animal were incubated for $90 \mathrm{~min}$ at room temperature (RT) in a phosphate buffer containing ${ }^{[125 I]}$ PE2I $(100 \mathrm{pM})$ as previously described (21). Nonspecific binding was assessed on adjacent sections with the same buffer containing $1 \mu \mathrm{M}$ cocaine (Cooper, France) and subtracted from total binding to yield specific binding. After rinsing and drying, the sections were exposed to sensitive films (Biomax MR, Kodak, France) with radioactive standard scales ( ${ }^{125} \mathrm{I}$-microscales, Amersham Bioscience $\mathrm{AB}, \mathrm{UK})$. The films were analyzed through an image analyzer (Betavision, Biospace instruments, France) after identifying the subtantia nigra pars compacta $(\mathrm{SNpc})$ and striatum areas. The absorbance obtained was converted into apparent tissue ligand concentrations with reference to standards and specific activity of the radioligand $(74 \mathrm{TBq} / \mathrm{mmol})$. The intensity of $\left[{ }^{125} \mathrm{I}\right]$ PE2I binding was expressed in $\mathrm{nCi} / \mathrm{mg}$ of equivalent tissue (mean $\pm \mathrm{SD}$ ).

Tyrosine hydroxylase immunostaining. The sections used were from the same animals analyzed for DAT autoradiographies. The slides were thawed, postfixed in $4 \%$ paraformaldehyde and the endogenous peroxidases were blocked. The primary antibody (rabbit polyclonal anti-TH antibody, Pel Freeze, USA, 1:500, 3h, RT), the secondary biotinylated antibody (goat anti rabbit, 1h, RT) and the Avidin Biotin Complex (Vector Laboratory, Burlingame, USA, $30 \mathrm{~min}$, RT) were consecutively incubated. The staining was revealed with diaminobenzidine (Sigma Chemical Co., France, 5 min). Internal control staining was performed by omitting the primary antibody. The optic density expressed as means \pm SD in arbitrary unit (AU) and the dopaminergic neurons counts were evaluated with an optical microscope (Leica, France) coupled to an image analyzer (Histolab, Microvision instruments, France) on 4 sections/animal. The neurons were counted in the SNpc, excluding the ventral tegmental area. The number of $\mathrm{TH}$ positive neurons is expressed as the total number of neurons $\pm \mathrm{SD}$.
DA content measurement. Both striata dissected from the same animal (3 groups: control, MPTP, LMT-MPTP in the same conditions as above; $n=5$ per group) were pooled and homogenized with $750 \mu \mathrm{L}$ ice-cold $0.12 \mathrm{M}$ perchloric acid $\left(0.1 \mathrm{mM}\right.$ EDTA, $\left.0.5 \mathrm{mM} \mathrm{Na}_{2} \mathrm{~S}_{2} \mathrm{O}_{5}\right)$. After centrifugation $\left(4^{\circ} \mathrm{C}\right.$, $16000 \mathrm{~g}, 20 \mathrm{~min}$ ), a fraction of the resulting supernatant was injected into the HPLC device (Beckman-Coulter, Fullerton, CA). The biogenic amines were separated on an Ultrasphere, Ion Pair, C18 Reverse Phase analytical column (LC-22C, BAS, West Lafayette, USA). The electrodetection was performed with a $+0.74 \mathrm{~V}$ electrochemical detector (Concorde, Waters, France). The mobile phase contained $20 \mathrm{mM}$ citric acid, $10 \mathrm{mM} \mathrm{Na} \mathrm{HPO}_{4}, 3.25 \mathrm{mM}$ heptane sulfonic acid, $3 \mathrm{mM}$ octane sulfonic acid, $0.1 \mathrm{mM}$ EDTA, $2 \mathrm{Mm} \mathrm{KCl}$, $0.2 \%$ diethylamine and $0.6 \%$ phosphoric acid, $7 \%$ acétonitrile, $3 \%$ methanol. The proteins from each sample were evaluated on the pellet with spectrophotometry according to Bradford (22). The results are expressed as means \pm SD in $\mathrm{nmol} \mathrm{DA} / \mathrm{mg}$ protein.

Active caspase 3 immunostaining. For active caspase 3 staining, the mice (3 groups: control, MPTP, LMT-MPTP in the same conditions as above; $n=$ 5 per group) were killed by cervical dislocation $7 \mathrm{~d}$ after MPTP intoxication. The brains were removed, postfixed in $4 \%$ paraformaldehyde, embedded in paraffin and cut into $16 \mu \mathrm{m}$ serial coronal sections. After deparafinization and rehydration, the sections were heated in a microwave oven $(750 \mathrm{~W} 1 \mathrm{~min}$, $300 \mathrm{~W} 9 \mathrm{~min}$ ) and cooled at RT. After saturation with goat serum, the primary antibody (rabbit polyclonal CM1 antibody, Dakocytomation, USA, 1:500, overnight, $37^{\circ} \mathrm{C}$ ), the secondary antibody (biotinylated rabbit anti mouse IgG 1\%, Dakocytomation, USA, 45 min, RT) and the Avidin Biotin Complex (Vector Laboratory, Burlingame, USA, $45 \mathrm{~min}, \mathrm{RT}$ ) were consecutively incubated and the staining revealed with diaminobenzidine (Sigma Chemical Co., France, $60 \mathrm{~min}$ ). Internal control staining was performed by omitting the primary antibody. The caspase- 3 positive cells were counted in the striatum and the results were expressed as mean \pm SEM. This experiment was performed on control, MPTP and LMT mice sections using 8 sections/animal.

Statistical analysis. Data were prospectively collected and analyzed with the Winks software (Texasoft, USA). Statistical significance was evaluated with the nonparametric Mann-Whitney test for nonpaired values from small groups. Statistical significance was considered for $p<0.05$.

\section{RESULTS}

MPTP and anticonvulsant treatment tolerance. Preliminary experiments were performed to monitor the impact of each anticonvulsant on mice growth and survival. Each of the 4 groups (MPTP, VPA-MPTP, TOP-MPTP and LMT-MPTP) was compared with the control group. During the observation

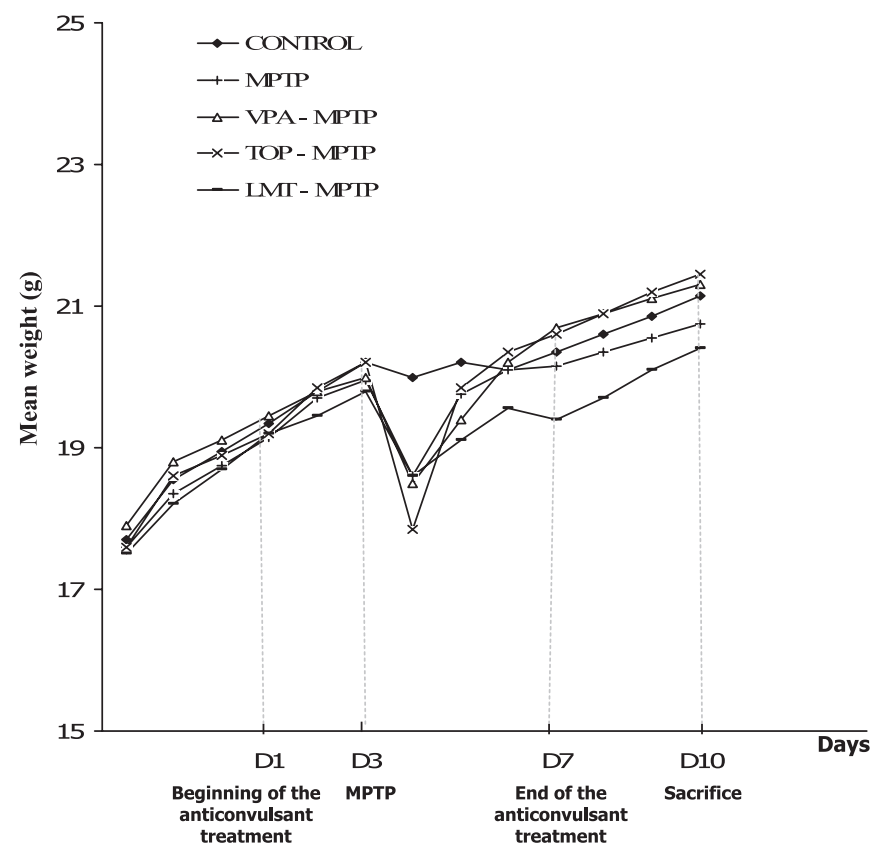

Figure 1. Mice growth curves during MPTP intoxication and anticonvulsant treatments. The mean weight per group ( $n=6$ in each group - C57/B16 strain) is indicated at each time point. 
period, all the animals survived and the MPTP-induced transient weight loss at day 4 did not significantly affect the final animal weight as shown in Figure 1 (MPTP: $20.75 \pm 0.42 \mathrm{~g}$; VPA: $21.3 \pm 0.67$ g; TOP: $21.45 \pm 0.52$ g; LMT: $20.4 \pm$ $0.36 \mathrm{~g}$ ). The brain mean weight was $0.4 \pm 0.02 \mathrm{~g}$ in all groups. To validate the reproducibility of the anticonvulsant oral administration procedure, we determined residual serum VPA concentration that, unlike LMT and TOP, is available on a routine basis (concentration $12 \mathrm{~h}$ after the last administration, $5 \mathrm{~d}$ of treatment, $n=5$ animals). The mean concentration was $1.94 \pm 0.95 \mathrm{mg} / \mathrm{L}$.

Effect of VPA, TOP and LMT on DAT density. Figure $2 A$ shows representative striatum autoradiographies of each mice group with an apparent normal DAT density in the LMTMPTP group. The DAT level, evaluated with the specific radio-labeled ligand $\left[{ }^{125} \mathrm{I}\right] \mathrm{PE} 2 \mathrm{I}$ reached $7.23 \pm 1.01 \mathrm{nCi} / \mathrm{mg}$ in the MPTP-intoxicated mice compared with $14.11 \pm 1.51$ $\mathrm{nCi} / \mathrm{mg}$ in the control group $(p=0.008)$. This significant reduction found in the striatum is shown on Figure $2 B$. VPA $(7.64 \pm 2.67 \mathrm{nCi} / \mathrm{mg})$ and TOP $(8.06 \pm 1.54 \mathrm{nCi} / \mathrm{mg}) \mathrm{did}$ not modify the DAT density versus the MPTP-intoxicated mice. In contrast, the DAT level in the LMT-MPTP group (13.98 \pm $1.34 \mathrm{nCi} / \mathrm{mg}$ ) was comparable to the control group but statistically higher than in the MPTP intoxicated mice $(p=0.008)$.

A
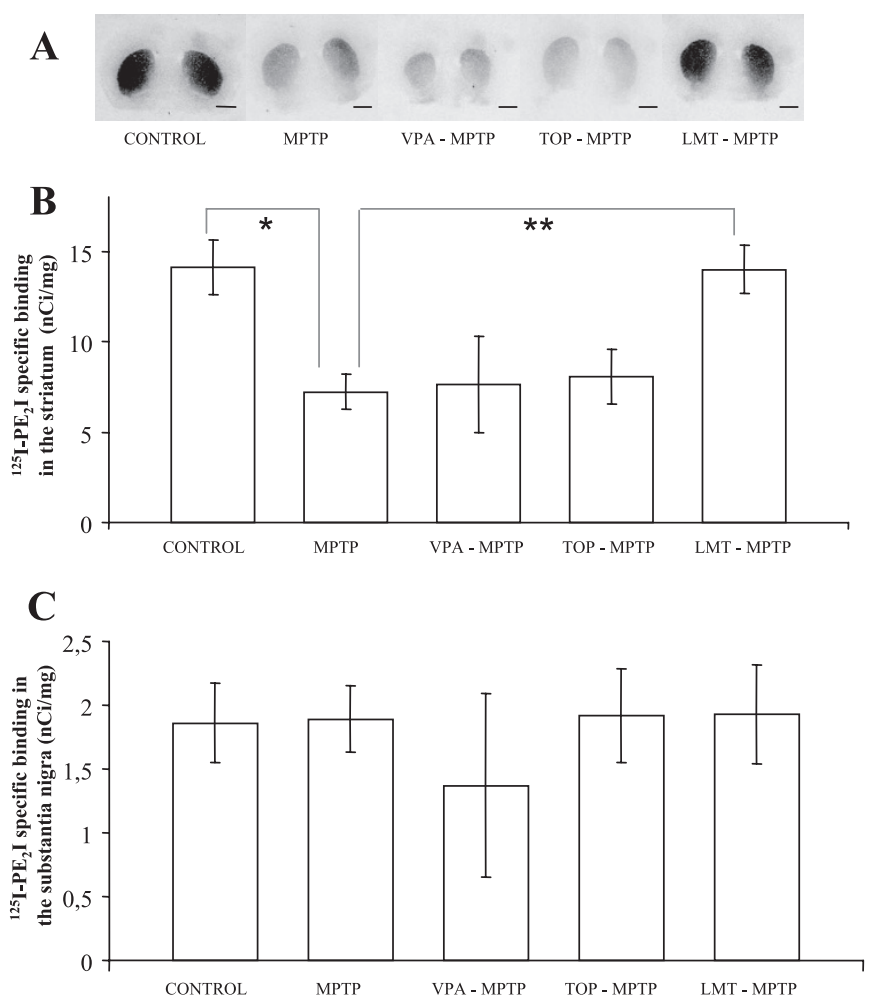

Figure 2. DAT density in control and MPTP intoxicated mice groups compared with the anticonvulsant treated groups ( $n=6$ in each group). (A) Representative striatum autoradiographies of each mice group showing a macroscopically apparent preservation of the basal level in the LMT-MPTP group. Interestingly, both external superior poles of the striata in the LMTMPTP group exhibit the highest signal intensity as in the basal physiologic pattern. Scale bar $=200 \mu \mathrm{m}$. Mean values $\pm \mathrm{SD}$ are expressed in $\mathrm{nCi} / \mathrm{mg}$ in the striatum $(B)$ and the $\mathrm{SN}(C)$. Noteworthy, DAT density is reduced in the MPTP group $(* p<0,01$ vs control level) and apparently normal in the LMT-MPTP group $(* * p<0,01 v s$ MPTP level).
As shown in Figure $2 C$, the DAT expression was not significantly reduced in the SNpc of MPTP-intoxicated mice $(1.89 \pm 0.26 \mathrm{nCi} / \mathrm{mg})$ compared with control mice (1.86 \pm $0.31 \mathrm{nCi} / \mathrm{mg})$.

Effect of VPA, TOP and LMT on TH immunoreactivity. The TH immunoreactivity was analyzed by optic density measurement in the striatum and the SNpc and subsequently confirmed in the SNpc by dopaminergic neurons count.

In the striatum, the TH immunoreactivity was significantly reduced in the MPTP group (91 $\pm 21 \mathrm{AU})$ versus the control group (164 $\pm 42 \mathrm{AU}, p=0.005)$. Compared with the MPTP group, this reduction was significantly blocked by the LMT treatment $(140 \pm 34 \mathrm{AU}, p=0.008)$, but not by VPA (79.5 $\pm 31 \mathrm{AU})$ or TOP $(77 \pm 12 \mathrm{AU})$ as shown in Figure $3 \mathrm{~A}$.

The TH immunostaining in the SNpc was statistically different between the MPTP intoxicated mice $(68 \pm 16 \mathrm{AU})$ and the control animals $(112 \pm 20 \mathrm{AU}, p=0.005)$. It remained at
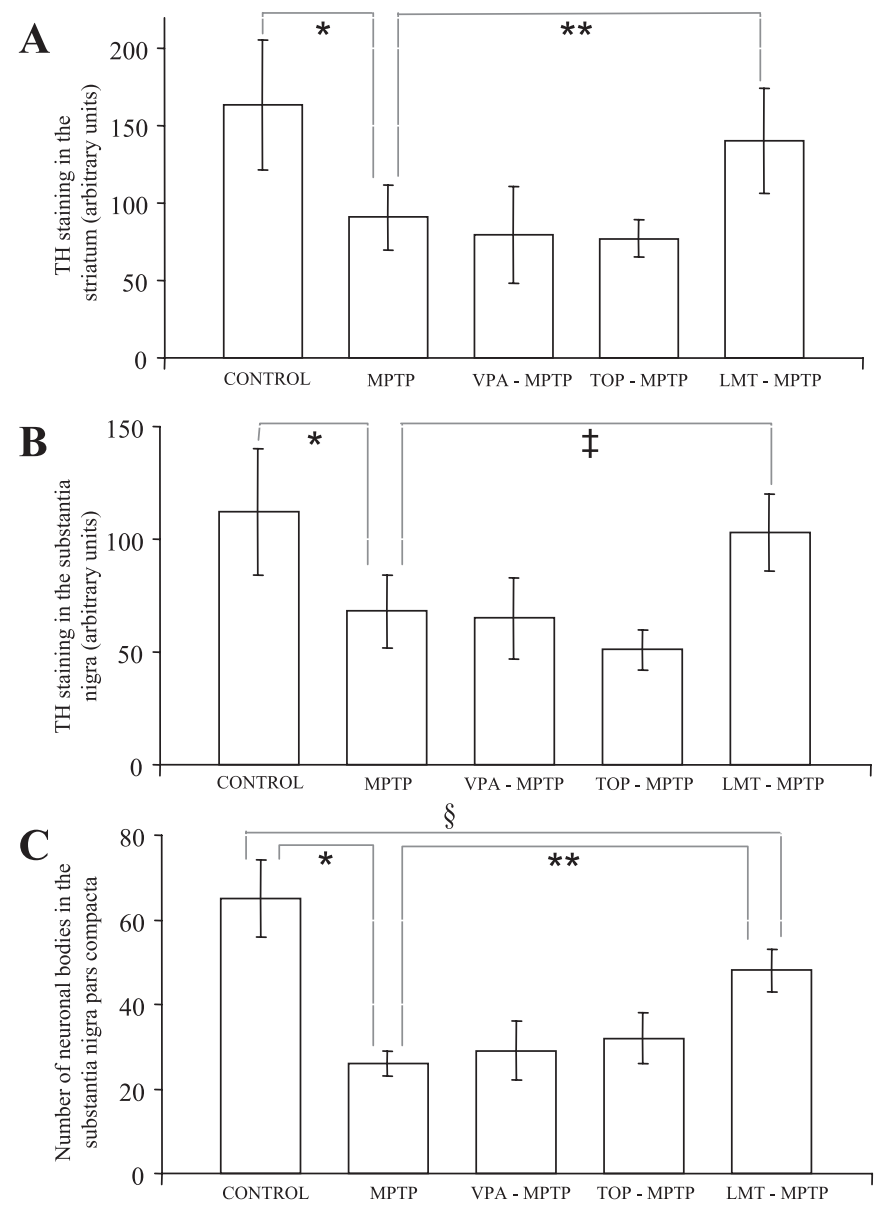

Figure 3. Effects of VPA, TOP and LMT on the TH immunostaining in the striatum and SN of C57/B16 MPTP intoxicated mice compared with control animals ( $n=6$ in each group). Each histogram represents the mean $\pm \mathrm{SD}$ of optic density in the striatum $(A)$ and in the $\mathrm{SN}(B)$. In both areas, TH immunoreactivity is significantly reduced in the MPTP intoxicated group $\left({ }^{*} p=0.005 v s\right.$ control group) and apparently protected in the LMT-MPTP group (** $p<0.01 v s$ MPTP group in the striatum, $\neq p=0.005 v s$ MPTP group in the SN). $C$ : Effects of VPA, TOP and LMT on the MPTP-induced dopaminergic cell loss. The number of TH positive neurons was significantly reduced after MPTP intoxication ( ${ }^{*} p=0.005 v s$ control group) but partially preserved in the LMT-MPTP group $(* * p<0.01 v s$ MPTP group; $\$ p<0,01$ $v s$ control group). 
a low level in the VPA and the TOP treated mice $(65 \pm 18 \mathrm{AU}$ in both groups) and was still significantly reduced versus control mice $(p=0.005)$. In contrast, the LMT treatment provided an apparent stability of the TH immunoreactivity $(103 \pm 17 \mathrm{AU})$. This level was higher than the one measured in the MPTP group $(p=0.005)$. The results are shown in Figure $3 B$.

The neurons count in the SNpc confirmed the optic density measurements: the number of TH positive neurons in the MPTP group (26 \pm 3 ) was significantly lower than in the control group $(65 \pm 9, p=0.005)$. It was comparable in the VPA-MPTP and TOP-MPTP groups versus the MPTP group $(29 \pm 7$ and $23 \pm 5$ neurons respectively) keeping lower than in the control group $(p=0.005)$. In the LMT-MPTP group, the number of TH positive neurons $(48 \pm 5)$ was higher than in the MPTP group $(p=0.008)$. These results are shown in Figure $3 C$. Representative microphotographs of the TH immunoreactivity are shown in the striatum (Fig. 4A) and the SNpc of control mouse brain (Fig. 4B). Higher magnification was used for the neurons count (Fig. $4 C$ ).

Effect of LMT on DA content. MPTP intoxication caused a significant reduction in striatal DA content compared with the controls (MPTP: $0.88 \pm 0.42 \mathrm{nmol} / \mathrm{mg}$ protein, control: $3.43 \pm 0.83 \mathrm{nmol} / \mathrm{mg}$ protein, $p=0.012$ ). In the LMT-MPTP group, the DA concentration was intermediary between the MPTP-intoxicated and control mice $(1.98 \pm 0.54 \mathrm{nmol} / \mathrm{mg}$ protein, $p=0.022$ versus both MPTP and control).

Effect of LMT on MPTP induced apoptosis. MPTPinduced apoptosis was investigated with activated caspase 3 immunostaining in the control, MPTP and LMT-MPTP groups. No staining was seen in the control and the LMTMPTP groups, whereas apoptosis was identified in the MPTP intoxicated mice $(0.67 \pm 0.27$ neurons, $p=0.0002)$. Representative microphotograph of active neuronal Caspase $3 \mathrm{im}$ munoreactivity is shown in Figure $4 D$.

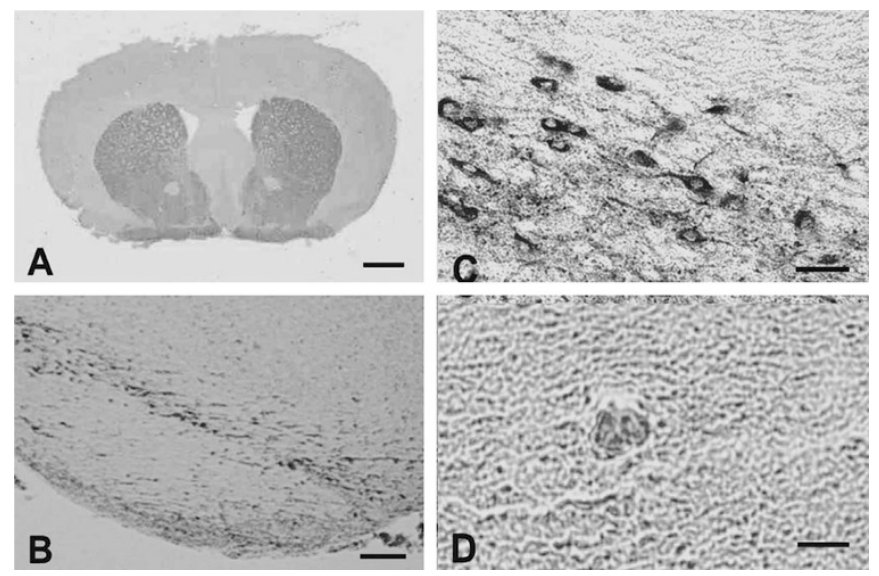

Figure 4. Representative microphotographs in the striatum $((A) \times 2.5$, scale bar: $500 \mu \mathrm{m})$ and the SNpc of a control mouse brain section showing regular neuronal TH immunoreactivity $((B) \times 10$, scale bar: $200 \mu \mathrm{m} ;(C) \times 20$, scale bar: $50 \mu \mathrm{m}) .(D)$ Representative microphotograph of active neuronal caspase-3 immunoreactivity from an MPTP intoxicated mice SNpc $(\times 40$, scale bar: $10 \mu \mathrm{m})$.

\section{DISCUSSION}

This study aimed to characterize the potential neuroprotective effects of VPA, TOP and LMT in the MPTP mouse model. MPTP inhibits the mitochondrial respiratory chain complex I and therefore provides, to some extent, a valuable tool to mimic the energy failure which occurs in various childhood conditions. The neuroprotective properties of these anticonvulsants were evaluated through the DAT density, the TH and Caspase-3 immunoreactivities and the DA content within the nigro-striatal network.

MPTP induced a weight loss in all intoxicated groups, with a complete retrieval within a day. This might be caused by the multiple manipulations on D3 or by a direct MPTP effect (23).

In the MPTP-intoxicated mice striatum, the DAT expression was reduced to $51 \%$ of the control levels. A similar decrease was observed in other studies using comparable methods $(24,25)$. In contrast, the DAT levels in the SN of the control and MPTP mice were not statistically different. Consequently, the anticonvulsant treatment impact on DAT density was not assessable in this area. This result is consistent with previous studies from our laboratory and others $(6,26)$ and probably relies on the autoradiographies spatial resolution, which prevents discrete evaluation of small structures such as the SNpc.

Through OD measurement, we found a $45 \%$ TH immunoreactivity decrease in the striatum and a $40 \%$ decrease in the $\mathrm{SNpc}$ of the MPTP intoxicated mice. Our results are consistent with the literature (27) reporting a $45 \%$ decrease in these experimental conditions in mice. Dopaminergic neurons count in the SNpc showed an even greater decrease (60\%) responsible for a residual neuronal count in the MPTP group, which represents $40 \%$ of the control level. The fact that the $\mathrm{TH}$ projections decrease in the striatum is lower than the dopaminergic neuronal death in the SNpc might reflect a compensatory $\mathrm{TH}$ over-expression in the remaining dopaminergic neurons through reactive sprouting. Such sprouting following MPTP has been already shown (26).

VPA enhances GABA release, inhibits sodium channels and stabilizes cell membranes (15). In accordance with a previous report (19), VPA did not provide neuroprotection in our study.

TOP carries anti-glutamatergic and pro-GABA properties, it inhibits calcium and sodium channels and it stabilizes cytoplasmic membranes $(14,16)$. This anticonvulsant did not show neuroprotective effects in our study and even seemed deleterious concerning TH immunoreactivity. Moreover, astrocytes exposed to TOP release higher reactive oxygen species (28). Therefore, both TOP and MPTP can induce oxidative stress and this might explain the absence of neuroprotection in our experimental conditions. As far as we know, these results are original since TOP has never been tested in the MPTP mouse model.

LMT is a potent anti glutamatergic anticonvulsant with calcium channels inhibition properties. It also inhibits sodium channels and stabilizes neuronal plasmic membranes. In this study, LMT provided a neuroprotective effect in the MPTP mouse model. Jones-Humble has shown that a single $40 \mathrm{mg}$ LMT dose, $2 \mathrm{~h}$ before MPTP intoxication, maintains normal 
DA striatal levels (20). Our results, based on DAT, TH, DA content and apoptosis analysis, confirm the LMT neuroprotective effect using complementary approaches which precise its mode of action.

LMT provided an apparent protection of DAT density in MPTP mice. These results could be explained by a striatal sprouting of the dopaminergic dendrites where DAT is highly expressed (29). After MPTP intoxication, a natural sprouting occurs and concerns $10 \%$ of the dendrites $(26,30)$. Thus, the near normal DAT level (99\%) found in the LMT-MPTP mice (versus $51 \%$ in the MPTP group) is probably related to the anticonvulsant treatment and not only spontaneous. This result suggests that LMT promotes dopaminergic neurons sprouting in the striatum following energetic stress.

The residual dopaminergic neurons count, evaluated through TH immunoreactivity in the SNpc, was significantly higher (74\% of the control levels) in the LMT-MPTP group than in the MPTP group (40\%). Previous studies have shown the absence of spontaneous dopaminergic neurons recovery or recruitment after MPTP lesion $(30,31)$. Therefore, the present results indicate that, under our experimental conditions, LMT significantly limited neuronal death in the SNpc. Our active caspase 3 staining study shows that this protection might occur through apoptosis inhibition.

Altogether, these data suggest that LMT limits dopaminergic neuronal death in the SNpc and promotes compensatory sprouting of the dendrites projections in the striatum.

In this model, the DA content decrease exceeds the neuronal loss due to MPTP-induced TH nitration (32). Conversely, such data indicate that TH phenotype rescue does not necessarily imply an active DA synthesis and, therefore, does not represent a reliable neuroprotection marker. Thus, we also analyzed DA production following LMT treatment. DA concentration is significantly higher in the LMT-MPTP group (58\% of the control levels) than in the MPTP group (26\%). This demonstrates that LMT maintains an active TH enzyme activity and not only a TH phenotype, which, thereby, confirms its neuroprotective property. LMT does not interact with monoamine oxidase B (33) and cytochrome P450 (34). Thus it is unlikely that LMT protects against MPTP toxicity through an increased MPP + catabolism (9).

According to the working hypothesis of our study, several advances in the anticonvulsants mechanisms of action deserve to be outlined: VPA, which does not provide neuroprotection in experimental energetic stress conditions, does not act on the ATP dependant calcium channels (ADCC) (35). TOP, which apparently enhances dopaminergic cell death during energy deficiency, inhibits the L type ADCC but stimulates the P/Q, $\mathrm{N}$ and $\mathrm{R}$ types (36). Conversely, LMT, which appears neuroprotective, inhibits the $\mathrm{L}, \mathrm{P} / \mathrm{Q}, \mathrm{N}$ and $\mathrm{R}$ types ADCC (37). Altogether, these data suggest that a wide range of ADCC types inhibition allows a significant ATP consumption braking which appears to be neuroprotective in energetic stress conditions. Presumably, this also prevents calcium overload that could, subsequently, promote the excitotoxic cascade.

In addition to its ATP dependent ion channels modulation properties, LMT might promote mitochondrial stability through its anti oxidant (39), anti excitotoxic (40) and anti apoptotic effects (41). LMT might also carry additional properties on the mitochondrial membrane permeability and/or the respiratory chain complexes activity, which have not been reported yet. In the 3-nitro-propionate model, where the mitochondrial complex II is targeted, LMT improves the cellular energy status (38). The fact that LMT is neuroprotective in several respiratory chain complex inhibition models suggests that LMT could be beneficial in various types of mitochondrial stress.

In conclusion, LMT shows an appropriate combination of neuroprotective properties in the energy deficiency model generated with MPTP intoxication in young mice. LMT is a widely used and well-tolerated molecule in children, even under two years old (39). LMT could therefore represent an interesting adjuvant neuroprotective therapy to consider in energetic stress of the basal ganglia encountered in various childhood conditions.

Acknowledgments. The authors thank P. Evrard and D. Guilloteau for support; M-C. Furon and M. Briand for technical assistance on animal experiments; Z. Gulhan and L. Galineau for contributive support on HPLC-ED measurements; P. Meunier, E. Autret-Leca and G. Paintaud for pharmacological assistance on anticonvulsant preparation and serum dosage; L. Scwhendimann, L. Tabone and F. Fetissof for precious help on paraffin embedding and caspase- 3 immunohistochemistry; F-X. Griffon and B. Giraudeau for expert contribution to the statistical analysis.

\section{REFERENCES}

1. Saudubray J-M, Charpentier C 2001 Clinical phenotypes: diagnosis/algorithms. In: Scriver CR, Beaudet AL, Sly WS, Valle D (eds) The metabolic and molecular bases of inherited disease. Mcgraw-Hill, New York, pp 327-400

2. Groenendaal F, Benders MJ, de Vries LS 2006 Pre-wallerian degeneration in the neonatal brain following perinatal cerebral hypoxia-ischemia demonstrated with MRI. Semin Perinatol 30:146-150

3. Okun JG, Horster F, Farkas LM, Feyh P, Hinz A, Sauer S, Hoffmann GF, Unsicker K, Mayatepek E, Kolker S 2002 Neurodegeneration in methylmalonic aciduria involves inhibition of complex II and the tricarboxylic acid cycle and synergistically acting excitotoxicity. J Biol Chem 277:14674-14680

4. Cavanagh JB, Harding BN 1994 Pathogenic factors underlying the lesions in Leigh's disease. Tissue responses to cellular energy deprivation and their clinicopathological consequences. Brain 117:1357-1376

5. Sie LT, van der Knaap MS, Oosting J, de Vries LS, Lafeber HN, Valk J 2000 MR patterns of hypoxic-ischemic brain damage after prenatal, perinatal or postnatal asphyxia. Neuropediatrics 31:128-136

6. Browne SE, Bowling AC, MacGarvey U, Baik MJ, Berger SC, Mugit MM, Bird ED, Beal MF 1997 Oxidative damage and metabolic dysfunction in Huntington's disease: selective vulnerability of the basal ganglia. Ann Neurol 41:646-653

7. Beal MF 2000 Energetics in the pathogenesis of neurodegenerative diseases. Trends Neurosci 23:298-304

8. Schulz JB, Matthews RT, Henshaw DR, Beal MF 1996 Neuroprotective strategies for treatment of lesions produced by mitochondrial toxins: implications for neurodegenerative diseases. Neuroscience 71:1043-1048

9. Przedborski S, Jackson-Lewis V, Djaldetti R, Liberatore G, Vila M, Vukosavic S, Almer G 2000 The parkinsonian toxin MPTP: action and mechanism. Restor Neurol Neurosci 16:135-142

10. Matthews RT, Ferrante RJ, Klivenyi P, Yang L, Klein AM, Mueller G, KaddurahDaouk R, Beal MF 1999 Creatine and cyclocreatine attenuate MPTP neurotoxicity. Exp Neurol 157:142-149

11. Srivastava R, Brouillet E, Beal MF, Storey E, Hyman BT 1993 Blockade of 1-methyl-4-phenylpyridinium ion (MPP + ) nigral toxicity in the rat by prior decortication or MK-801 treatment: a stereological estimate of neuronal loss. Neurobiol Aging 14:295-301

12. Alexi T, Borlongan CV, Faull RL, Williams CE, Clark RG, Gluckman PD, Hughes PE 2000 Neuroprotective strategies for basal ganglia degeneration: Parkinson's and Huntington's diseases. Prog Neurobiol 60:409-470

13. Obrenovitch TP, Urenjak J 1998 Glutamate release inhibitors: a critical assessment of their action mechanism. Amino Acids 14:143-150 
14. Shank RP, Gardocki JF, Streeter AJ, Maryanoff BE 2000 An overview of the preclinical aspects of topiramate: pharmacology, pharmacokinetics and mechanism of action. Epilepsia 41:S3-S9

15. Löscher W 1999 Valproate: a reappraisal of its pharmacodynamic properties and mechanisms of action. Prog Neurobiol 58:31-59

16. White HS 2003 Mechanism of action of newer anticonvulsants. J Clin Psychiatry 64:5-8

17. Kudin AP, Debska-Vielhaber G, Vielhaber S, Elger CE, Kunz WS 2004 The mechanism of neuroprotection by topiramate in an animal model of epilepsy. Epilepsia 45:1478-1487

18. Yang Y, Li Q, Shuaib A 2000 Enhanced neuroprotection and reduced hemorrhagic incidence in focal cerebral ischemia of rat by low dose combination therapy of urokinase and topiramate. Neuropharmacology 39:881-888

19. Melamed E, Martinovits G, Pikarsky E, Rosenthal J, Uzzan A 1986 Diphenylhydantoin and phenobarbital suppress the dopaminergic neurotoxicity of MPTP in mice. Eur J Pharmacol 128:255-257

20. Jones-Humble SA, Morgan PF, Cooper BR 1994 The novel anticonvulsant lamotrigine prevents dopamine depletion in C57 black mice in the MPTP animal model of Parkinson's disease. Life Sci 54:245-252

21. Chalon S, Garreau L, Emond P, Zimmer L, Vilar MP, Besnard JP, Guilloteau D 1999 Pharmacological characterization of (E)-N-(3-iodoprop-2-enyl)-2beta-carbomethoxy-3beta-(4'-methylphenyl)n ortropane as a selective and potent inhibitor of the neuronal dopamine transporter. J Pharmacol Exp Ther 291:648-654

22. Bradford MM 1976 A rapid and sensitive method for the quantitation of microgram quantities of protein utilizing the principle of protein-dye binding. Anal Biochem 72:248-254

23. Ferger B, Spratt C, Earl CD, Teismann P, Oertel WH, Kuschinsky K 1998 Effects of nicotine on hydroxyl free radical formation in vitro and on MPTP-induced neurotoxicity in vivo. Naunyn Schmiedebergs Arch Pharmacol 358:351-359

24. D'Astous M, Morissette M, Di Paolo T 2004 Effect of estrogen receptor agonists treatment in MPTP mice: evidence of neuroprotection by an ER alpha agonist. Neuropharmacology 47:1180-1188

25. Kilbourn MR, Kuszpit K, Sherman P 2000 Rapid and differential losses of in vivo dopamine transporter (DAT) and vesicular monoamine transporter (VMAT2) radioligand binding in MPTP-treated mice. Synapse 35:250-255

26. Mitsumoto Y, Watanabe A, Mori A, Koga N 1998 Spontaneous regeneration of nigrostriatal dopaminergic neurons in MPTP-treated C57BL/6 mice. Biochem Biophys Res Commun 248:660-663

27. Araki T, Mikami T, Tanji H, Matsubara M, Imai Y, Mizugaki M, Itoyama Y 2001 Biochemical and immunohistological changes in the brain of 1-methyl-4-phenyl1,2,3,6-tetrahydropyridine (MPTP)-treated mouse. Eur J Pharm Sci 12:231-238
28. Pavone A, Cardile V 2003 An in vitro study of new antiepileptic drugs and astrocytes. Epilepsia 44:34-39

29. Jakowec MW, Nixon K, Hogg E, McNeill T, Petzinger GM 2004 Tyrosine hydroxylase and dopamine transporter expression following 1-methyl-4-phenyl-1,2,3,6tetrahydropyridine-induced neurodegeneration of the mouse nigrostriatal pathway. J Neurosci Res 76:539-550

30. Jackson-Lewis V, Jakowec M, Burke RE, Przedborski S 1995 Time course and morphology of dopaminergic neuronal death caused by the neurotoxin 1-methyl-4phenyl-1,2,3,6-tetrahydropyridine. Neurodegeneration 4:257-269

31. Kurosaki R, Muramatsu Y, Kato H, Araki T 2004 Biochemical, behavioral and immunohistochemical alterations in MPTP-treated mouse model of Parkinson's disease. Pharmacol Biochem Behav 78:143-153

32. Ara J, Przedborski S, Naini AB, Jackson-Lewis V, Trifiletti RR, Horwitz J, Hischiropoulos H 1998 Inactivation of tyrosine hydroxylase by nitration following exposure to peroxynitrite and MPTP. Proc Natl Acad Sci USA 95:7659-7663

33. Southam E, Pereira R, Stratton SC, Sargent R, Ford AJ, Butterfield LJ, Wheable JD, Beckett SR, Roe C, Marsden CA, Hagan RM 2005 Effect of lamotrigine on the activities of monoamine oxidases $\mathrm{A}$ and $\mathrm{B}$ in vitro and on monoamine disposition in vivo. Eur J Pharmacol 519:237-245

34. Anderson GD 1998 A mechanistic approach to antiepileptic drug interactions. Ann Pharmacother 32:554-563

35. Czapinski P, Blaszczyk B, Czuczwar SJ 2005 Mechanisms of action of antiepileptic drugs. Curr Top Med Chem 5:3-14

36. Zhang X, Velumian AA, Jones OT, Carlen PL 2000 Modulation of high voltage activated calcium channels in dentate granule cells by topiramate. Epilepsia 41:S52-S60

37. McGeer EG, Zhu SG 1990 Lamotrigine protects against kainate but not ibotenate lesions in rat striatum. Neurosci Lett 112:348-351

38. Lee WT, Shen YZ, Chang C 2000 Neuroprotective effect of lamotrigine and MK-801 on rat brain lesions induced by 3-nitropropionic acid: evaluation by magnetic resonance imaging and in vivo proton magnetic resonance spectroscopy. Neuroscience 95:89-95

39. Serteser M, Ozben T, Gumuslu S, Balkan S, Balkan E 2002 Lipid peroxidation in rat brain during focal cerebral ischemia: prevention of malondialdehyde and lipid conjugated diene production by a novel antiepileptic, lamotrigine. Neurotoxicology 23:111-119

40. Von Wegerer J, Hesslinger B, Berger M, Walden J 1997 A calcium antagonistic effect of the new antiepileptic drug lamotrigine. Eur Neuropsychopharmacol 7:77-81

41. McDonald DG, Najam Y, Keegan MB, Whooley M, Madden D, McMenamin JB 2005 The use of lamotrigine, vigabatrin and gabapentin as add-on therapy in intractable epilepsy of chidhood. Seizure 14:112-116 